\title{
Binaural release from temporal induction
}

\author{
MAKIO KASHINO and RICHARD M. WARREN \\ University of Wisconsin, Milwaukee, Wisconsin
}

\begin{abstract}
This study examined the effects of interaural phase difference (IPhD) relations in temporal induction (TI) - that is, the perceived continuity of a fainter sound (inducee) through an interrupting higher amplitude sound (inducer). The extent of TI was measured both directly as the upper amplitude level for continuity and indirectly as the loudness reduction of the inducer. It was found that TI was inhibited when the IPhD of the inducer and the IPhD of the inducee differed. It was also found that the extent of induction was positively related to the masking potential of the inducer as measured by the binaural masking level difference procedure. These results suggest that TI involves processes that are affected by interaural phase differences and that the masking potential rule, shown by previous studies to apply in the frequency domain, applies also in the IPhD domain. The present findings are consistent with the hypothesis that TI results from allocation of a portion of the neural excitation produced by the inducer over to the inducee if the inducer has the appropriate masking potential. As a consequence, obliterated signals can be selectively restored on the basis of both frequency-specific monaural cues and azimuth-related binaural cues, abilities that can enhance detection of signals under the noisy conditions of everyday life.
\end{abstract}

When a portion of a sound is deleted and replaced by higher amplitude sound, under appropriate conditions, listeners perceive the deleted sound as continuous and are unable to distinguish the perceptually synthesized segment from those actually present (for review, Warren, 1984). This perceptual synthesis of missing sounds, or temporal induction (TI), occurs with various types of acoustic signals, ranging from pure tones to speech, and plays an important role in reducing interference by extraneous sounds in the real world, where two or more sounds often overlap.

Previous studies have indicated that TI involves frequency-selective processes. Warren, Obusek, and Ackroff (1972) alternated a fixed-amplitude $1000-\mathrm{Hz}$ pure tone (the inducer) with pure tones of various frequencies (the inducees), with each sound lasting for $300 \mathrm{msec}$, and measured the continuity limits (i.e., the highest level of the inducees at which they were heard as continuous). They found that the frequency-dependent continuitylimit curve resembled the curve they obtained when the inducer was left on continuously, and the masked detection threshold of the pulsed inducee was determined. It was concluded that TI may occur if the inducer has a spectrum that could mask the inducee if they were present simultaneously. Houtgast (1972) also discovered the relationship between the limits for perceived continuity, or pulsation threshold (PT), and the masking potential of the higher amplitude sound in his project to demonstrate

This study was supported by Grant DC00208 from the National Institutes of Health to the second author. The valuable contributions of James A. Bashford Jr., Bradley S. Brubaker, Keri R. Riener, and Eric W. Healy are gratefully acknowledged. Correspondence should be addressed to $M$. Kashino, Information Science Research Laboratory, NTT Basic Research Laboratories, 3-1, Morinosato Wakamiya, Atsugi, Kanagawa 243-01, Japan. lateral suppression in hearing, and he also stated that, in order for continuity to occur, the neural activity evoked by the higher amplitude interrupter must include the activation of neural activity corresponding to the continuation of the lower amplitude signal.

It was also hypothesized (Warren et al., 1972) that TI involves the allocation of a portion of the neural activity produced by the inducer over to the inducee for synthesis of continuity, which should also result in a measurable decrease in the inducer's apparent amplitude or loudness under appropriate conditions. Recently, Warren, Bashford, Healy, and Brubaker (1994) reported experimental results that support this allocation hypothesis. They demonstrated that the loudness reduction of the inducer occurred at levels not only below but also above the pulsation threshold. They attributed this to the existence of illusory lengthening of the inducee above the continuity limit (or pulsation threshold): It was reported that, when the frequency separation between the tones representing the inducee and the inducer was increased to the point that continuity was no longer heard, a partial gap closure occurred as measured by an increase in the apparent duration of the inducee that was insufficient to bridge the gaps separating successive statements. This illusory lengthening was accompanied by a decrement in the inducer's apparent amplitude that was proportional to the increment in the inducee's apparent duration. Additionally, when a seemingly continuous inducee and the higher amplitude inducer were both $1000-\mathrm{Hz}$ tones, smaller level differences between the two produced larger decreases in the apparent amplitude of the inducer. These results are consistent with the hypothesis that a portion of the inducer's neural activity is subtracted from the representation of the inducer and used to produce the continuity of the inducee. 
In sum, TI can be thought of as the allocation of the frequency-selective neural activity. This makes sense if we consider the ecological function of TI. In the real world, the perceptual restoration of a segment is appropriate when the segment is actually present but masked by an extraneous sound. If the segment could not have been masked by the extraneous sound, then synthesizing the segment would be inappropriate. Thus, the spectral selectivity of TI helps the auditory system to determine whether or not the inducee could be present as masked component. The subtractive operation of $\mathrm{TI}$ is consistent with this approach, if we take into account the fact that the masking takes place when an extraneous sound is added to the target sound in the real world. Therefore, an accurate percept of the inducer's amplitude requires the subtraction of the inducee's contribution from the total neural activity.

Now a question arises: Are there factors other than the frequency and amplitude relations of sounds that determine whether or not TI occurs? Answering this question will promote understanding what kinds of processes are involved in TI and how the ecological functions are realized when such processes are used in dealing with the "cocktail party" phenomenon. In this study, we examine the spatial (interaural difference) selectivity of TI.

There are at least two reasons to anticipate a spatial selectivity for TI. First, it is physically possible for the interaural differences of acoustic signals - such as interaural time difference (ITD), interaural phase difference (IPhD), and interaural level difference (ILD), each of which is correlated with the spatial locations of the sound sources - to provide information concerning whether the inducee is present during the interruption by the inducer. Second, there is both psychophysical and physiological evidence that the auditory system has mechanisms that are sensitive to interaural disparity, which are used for sound source localization and signal detection (for review, Durlach \& Colburn, 1978; Yin \& Chan, 1988).

Among the many phenomena that show an advantage of binaural over monaural hearing, the effect most relevant to the present topic is the binaural masking level difference (BMLD). This refers to the improvement in detectability of a target signal presented with a masking sound when using two ears over the detectability using a single ear. The BMLD depends on the interaural differences in the target and masker and can be as large as $25 \mathrm{~dB}$ under optimum conditions (Durlach \& Colburn, 1978). The question addressed here is whether BMLD is correlated with TI, just as the spectrally dependent masking is. If the BMLD is related to TI, then the masking potential rule of TI theory is shown to hold in the spatial (interaural difference) domain as well as in the frequency domain. There have been two reports concerning the relationship between the BMLD and the pulsation threshold. One is an abstract by Soderquist (1981), and the other is Hartmann's (1984) study searching for evidence for central lateral inhibition. In Hartmann's more detailed study, he measured the pulsation threshold for tones with low-pass noise maskers under four types of IPhD combinations. It was observed that the pulsation thresholds were lower when IPhD of the tone and that of the noise were different (one being antiphasic and the other cophasic) than when they were identical. Though his results are basically consistent with our expectation, he did not provide direct measures of the quantitative relation between masking and continuity. In addition, he did not address the question of whether illusory continuity involves a reallocation of the neural activity that is dependent upon interaural differences as well as frequency differences (this point has not been investigated in studies involving the pulsation threshold paradigm).

The present study employs a temporal induction paradigm to provide information concerning the ecological functions, as well as the mechanisms employed for implementing the perceptual restoration of masked signals. This paper describes three experiments. Experiment 1 was designed to demonstrate the spatial selectivity of TI directly by examining whether the differences in interaural phase disparities exhibited by the inducer and the inducee affect the continuity limit. Experiment 2 was designed to examine the quantitative relationship between the continuity limit observed in Experiment 1 and the masked detection threshold of the inducee under binaural masking level difference conditions with the inducer serving as the masker. Finally, Experiment 3 was designed to see whether the loudness reduction of the inducer is affected by the differences in the interaural phase disparity between inducer and inducee. Taken as a set, Experiments 1 and 2 test the masking potential requirements, and Experiment 3 tests the amplitude reduction consequence of the allocation hypothesis for TI when extended to the binaural disparity domain.

In these three experiments, we used the four types of $\mathrm{IPhD}$ combinations employed conventionally in BMLD studies (the inducee [presented as the signal target] is designated by $\mathrm{S}$; the inducer [presented as the masker] is designated by $\mathbf{M}$; zero interaural phase is designated by $o$; antiphasic interaural phase is designated by $\pi$ ): (1) MoSo, (2) $\mathrm{M} \pi \mathrm{S} \pi$, (3) $\mathrm{M} \pi \mathrm{So}$, and (4) $\mathrm{MoS} \pi$.

\section{EXPERIMENT 1}

In Experiment 1, we measured the continuity limits with each of the IPhD combinations (MoSo, $\mathrm{M} \pi \mathrm{S} \pi, \mathrm{M} \pi \mathrm{So}$, and $\operatorname{MoS} \pi$ ). The listener's task was to judge whether the inducee appeared continuous at various inducee levels while the inducer level was fixed. The continuity limit was defined as the inducee level at which the listener's judgment changes from "continuous" to "discontinuous."

\section{Method}

Subjects. The 5 subjects were laboratory personnel having prior experience as listeners in psychophysical studies. The subjects participated in several hours of preliminary observations before the formal experiment.

Stimuli. The stimuli consisted of two sounds that were presented in an alternating fashion as is shown in Figure 1 . The inducee was a $500-\mathrm{Hz}$ sinusoidal tone, and the inducer was a $1 / 3$-octave noise 
band centered at $500 \mathrm{~Hz}$ ( $48 \mathrm{~dB} /$ octave slopes). The on time and off time for each sound were $200 \mathrm{msec}$, and the total duration of a stimulus was $3.4 \mathrm{sec}$. To reduce switching transients, signals were gated on and off with a $10-\mathrm{msec}$ raised cosine ramp. The ramps of adjacent statements were completely overlapped. Waveforms of the inducee or the inducer presented at the left and the right ears were identical $(\mathrm{IPhD}=0)$ or polarity-inverted $(\mathrm{IPhD}=\pi)$.

The stimuli were synthesized digitally using a numeric computation software package (Mathworks MATLAB) on a personal computer (Apple Macintosh II fx) and played out through a twochannel 16-bit $\mathrm{D} / \mathrm{A}$ converter with built-in antialiasing filters (Digidesign Audio Interface) at a $22.05-\mathrm{kHz}$ sampling rate. The outputs of the Audio Interface were amplified using an audio mixer (RAMSA WR 133) and delivered through circumaural headphones (Sennheiser HD 230).

The inducer was always presented at a sound pressure level of $70 \mathrm{~dB}$, and the inducee was presented at sound pressure levels ranging from 35 to $70 \mathrm{~dB}$. Signal levels were measured using a sound level meter (Brüel and Kjær Model 2230) fitted with a flatplate coupler and operating in its slow-average mode. A-scale weighting was used throughout. The waveforms and spectra of the signals as transduced by the Sennheiser HD 230 headphones were examined using a signal analyzer (Hewlett-Packard 3561 A).

Procedure. The continuity limits were measured using a modified method-of-limits procedure. On the beginning trial of an ascending series of judgments, the inducee was presented at a low sound pressure level--varying randomly from 35 to $40 \mathrm{~dB}$-and increased regularly in steps of $2 \mathrm{~dB}$ for successive trials until the listener detected discontinuity in the inducee. For a descending series of trials, the inducee started at a level ranging from 65 to $70 \mathrm{~dB}$ and then decreasing in 2-dB steps until the listener judged the inducee to be completely continuous. The intertrial interval was $3 \mathrm{sec}$. For each IPhD condition, the listener was presented alternately with 10 ascending and 10 descending series of trials, for a total of 20 series. For each IPhD condition, half of the 20 series used evennumbered sound pressure levels (e.g., $70 \mathrm{~dB}, 68 \mathrm{~dB}, 66 \mathrm{~dB}, \ldots$ ), and the rest used odd-numbered levels (e.g., $69 \mathrm{~dB}, 67 \mathrm{~dB}$, $65 \mathrm{~dB}, \ldots)$. The 20 series were divided into two blocks, one of which consisted of 10 even-numbered ascending series and 10 oddnumbered descending series, and the other of which consisted of 10 even-numbered descending series and 10 odd-numbered ascending series. The average of the 20 series for each $\mathrm{IPhD}$ condition was taken as a continuity limit.

\section{Results and Discussion}

Figure 2 shows the mean continuity limits under the four IPhD conditions. In MoSo and $\mathrm{M} \pi \mathrm{S} \pi$ conditions

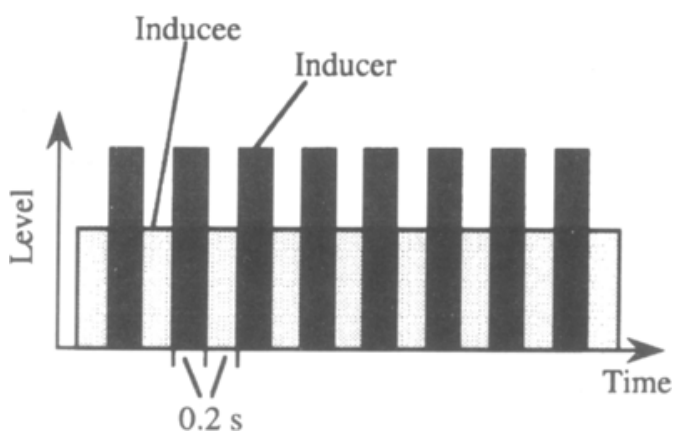

Figure 1. Stimuli employed for measuring the continuity limits of a $500-\mathrm{Hz}$ sinusoidal inducee alternating with a $500-\mathrm{Hz}, 1 / 3-$ octave band noise inducer. The interaural phase difference combinations of the inducee and the inducer were $M o S o, M \pi S \pi$, $\operatorname{MoS} \pi$, and $M \pi S o$ (see text for further description).

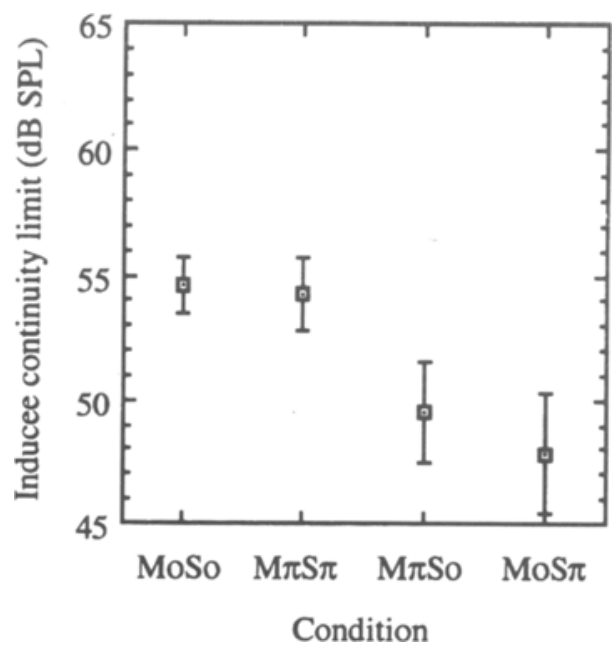

Figure 2. Continuity limits of a $500-\mathrm{Hz}$ sinusoidal inducee alternating with a $500-\mathrm{Hz}, 1 / 3$-octave band noise inducer under four types of interaural phase differences. Plotted values are means and standard errors for 5 listeners.

where IPhDs of the inducee and the inducer were identical (we will call these conditions isophasic IPhD conditions), the continuity limits were between 54 and $55 \mathrm{~dB}$ SPL. The continuity limits were about 5-7 dB lower in $\mathrm{M} \pi$ So and $\operatorname{MoS} \pi$ conditions where IPhDs of the inducee and the inducer were different (heterophasic IPhD conditions). A repeated measure analysis of variance (ANOVA) yielded that an effect of IPhD conditions was statistically significant $[F(3,12)=18.45, p<.0005]$. Evidence of the observed difference between the isophasic and heterophasic IPhD conditions was obtained in subsequent, single- $d f$ contrast, which compared the mean of the two isophasic IPhD conditions with the mean of two heterophasic IPhD conditions $[F(1,12)=$ $53.15, p<.0005]$.

These results indicate that IPhD relations of the inducee and the inducer affect the TI, even when the spectral masking potential and other monaural conditions are kept constant. From the ecological viewpoint, it makes sense that $\mathrm{TI}$ occurs more readily for the isophasic $\mathrm{IPhD}$ conditions than for the heterophasic IPhD conditions, because the difference in IPhD between the inducee and the inducer makes the discontinuity of the inducee during the interruption explicit. The results are also consistent with the hypothesis that neural processing responsible for detection of interaural phase (and/or time) differences are involved in TI.

\section{EXPERIMENT 2}

The results of Experiment 1 demonstrated that TI was affected by the IPhD relationship between the inducer and the inducee. In Experiment 2, we measured binaural signal detection threshold in the presence of masking noise using the same subjects and IPhD conditions as in Experiment 1, in order to examine the relationship between TI and the binaural masking. The question ad- 


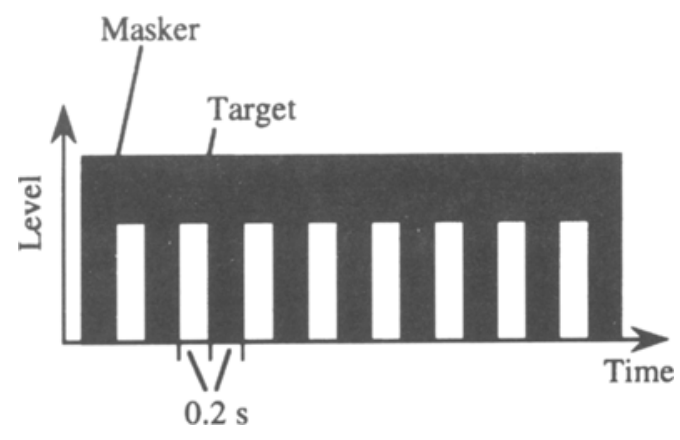

Figure 3. Stimuli employed for measuring the binaural signal detection thresholds of a $500-\mathrm{Hz}$ sinusoidal target in the presence of a 500-Hz, 1/3-octave band noise masker. The four types of interaural phase difference combinations of the target and the masker were MoSo, $M \pi S \pi, M o S \pi$, and $M \pi S o$ (see text for further description).

dressed was whether the masking potential rule holds in $\mathrm{IPhD}$ domain as well as in the frequency domain.

\section{Method}

Subjects. The same 5 subjects as in Experiment 1 participated in this experiment.

Stimuli. The stimuli consisted of a discontinuous target signal and a continuous masker as is shown in Figure 3. The target was a $500-\mathrm{Hz}$ sinusoidal tone of which on time and off time were $200 \mathrm{msec}$. To reduce switching transients, the target signals were gated on and off with a 10 -msec raised cosine ramp. The masker was a $1 / 3$-octave noise band centered at $500 \mathrm{~Hz}(48 \mathrm{~dB} /$ octave slopes), which was on for $3.4 \mathrm{sec}$. Waveforms of both the target and the masker presented at the left and the right ears were identical $(\mathrm{IPhD}=0)$ or polarity-inverted $(\mathrm{IPhD}=\pi)$, giving rise to the four conditions: MoSo, $\mathrm{M} \pi \mathrm{S} \pi$, $\mathrm{M} \pi \mathrm{So}$, and $\mathrm{MoS} \pi$.

The masker was always presented at a sound pressure level of $70 \mathrm{~dB}$, and the target was presented at sound pressure levels ranging from 39 to $72 \mathrm{~dB}$. The same apparatus as that in Experiment 1 was used for the synthesis and the presentation of the stimuli.

Procedure. The binaural signal detection thresholds were measured using a modified method-of-limits procedure. The listener's task was to judge whether he or she detected a target at various target levels while the masker level was fixed. For the beginning trial of an ascending series, the target was presented at a low sound pressure level varying randomly from 39 to $44 \mathrm{~dB}$ and increased in 2-dB steps until the listener detected the target. For a descending series, the masker started at a level varying randomly from 67 to $72 \mathrm{~dB}$ and then decreased in 2-dB steps until the listener was not able to detect the target. As in Experiment 1, there were two blocks, one of which consisted of 10 even-numbered ascending series and 10 odd-numbered descending series, and the other of which consisted of 10 even-numbered descending series and 10 odd-numbered ascending series. The average of the 20 series for each $\mathrm{IPhD}$ condition was taken as a detection threshold.

\section{Results and Discussion}

Figure 4 shows the mean signal detection thresholds for the four IPhD conditions. In the isophasic IPhD conditions (MoSo and $\mathrm{M} \pi \mathrm{S} \pi$ ), the detection thresholds were approximately $62 \mathrm{~dB}$. On the other hand, the detection threshold was about $54 \mathrm{~dB}$ in $\mathrm{M} \pi$ So condition and about $51 \mathrm{~dB}$ in $\mathrm{MoS} \pi$ condition, representing BMLDs of about 8 and $11 \mathrm{~dB}$, respectively. This overall tendency resembled the continuity limits, though the BMLD was larger than the difference in the continuity limits between the isophasic and heterophasic IPhD conditions.

The effect of IPhD conditions on the detection thresholds was statistically significant. The five listeners' mean thresholds were subjected to a repeated measure ANOVA, which yielded a significant effect $[F(3,12)=50.35, p<$ $.0005]$. The difference between the isophasic and heterophasic IPhD conditions was also significant in a single- $d f$ contrast, which compared the mean of the two isophasic $\mathrm{IPhD}$ conditions with the mean of the two heterophasic IPhD conditions $[F(1,12)=146.41, p<.0005]$.

These results, along with the results from Experiment 1, indicate that the masking potential rule applies in the IPhD domain as well as in the frequency domain. However, it should be noted that the masking potential is not the sole determinant for TI, because the detection thresholds were higher than the continuity limits by about $4-8 \mathrm{~dB}$ on average for the 5 subjects. A two-factor (continuity/masking, IPhD conditions) repeated measure ANOVA approached significance in the continuity/masking effect $[F(1,4)=7.267$, $p<.06]$. Therefore, single- $d f$ contrasts were performed to compare the data obtained for continuity and for masking after collapsing the data for the two isophasic (or heterophasic) conditions. The differences between continuity and masking were significant both in the isophasic conditions $[F(1,12)=72.11, p<.0005]$ and in the heterophasic conditions $[F(1,12)=20.07, p<.001]$. Similar quantitative differences between masked thresholds and continuity limits were reported for diotic conditions when a $1000-\mathrm{Hz}$ pure tone was alternated with a $1 / 3$-octave noise band centered at $1000 \mathrm{~Hz}$ (Warren et al., 1972).

\section{EXPERIMENT 3}

The results from Experiments 1 and 2 suggest that TI involves $\mathrm{IPhD}$-selective processes. If $\mathrm{TI}$ is a reallocation

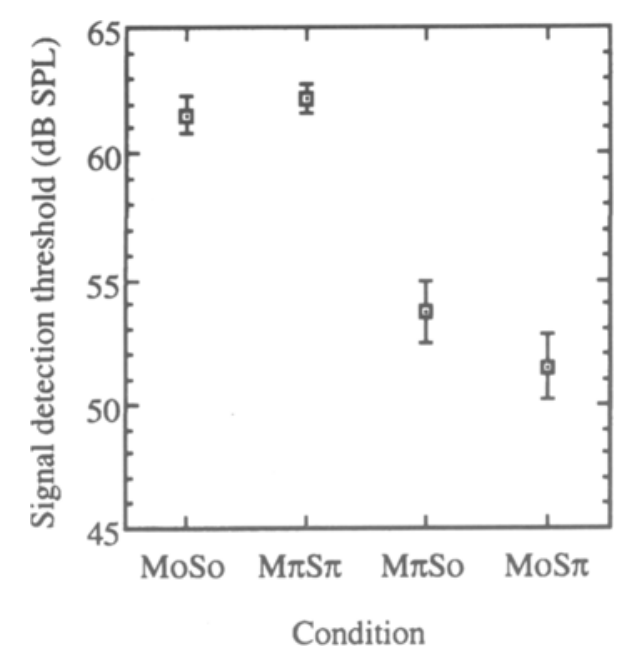

Figure 4. Binaural signal detection thresholds of a $500-\mathrm{Hz}$ sinusoidal target in the presence of a $500-\mathrm{Hz}, 1 / 3-$ octave band noise masker under four types of interaural phase difference combinations. Plotted values are means and standard errors for 5 listeners. 
of the IPhD-selective neural activity evoked by the inducer, it is to be expected that the loudness of the inducer is reduced when the inducee is perceived continuously, as was shown earlier with diotic presentation (Warren et al., 1994), and further that the amount of the reduction depends on IPhD relations between the inducee and the inducer. Experiment 3 was designed to test this prediction by measuring loudness of the inducer under the four types of IPhD conditions.

\section{Method}

Subjects. The same 5 subjects as in Experiments 1 and 2 participated in this experiment.

Stimuli. The stimuli consisted of an induction stimulus and a comparison stimulus as shown in Figure 5. The induction stimulus consisted of two alternating sounds, differing only in intensity. Both sounds were $200-\mathrm{msec}, 500-\mathrm{Hz}$ pure tones. The choice of inducer was different from that in Experiments 1 and 2. The reason is as follows. In Experiments 1 and 2, the main purpose was to compare continuity limits and masked thresholds directly. In Experiment 2, a $500-\mathrm{Hz}$ tone could not be used as the masker, since when the two tones were combined, a cophasic target would increase the level of the masker, while an antiphasic target would reduce its level, transforming the task to a just-noticeable difference discrimination of amplitude. Therefore, we used 1/3-octave band of noise along with a tone in both experiments - as a masker in Experiment 2 and as an inducer in Experiment 1. On the other hand, the purpose of Experiment 3 was to demonstrate loudness reduction of the inducer. According to the allocation theory, the loudness reduction is expected to be the largest when the neural excitation pattern of the inducee and that of the inducer are as similar as possible. This expectation was confirmed by preliminary observation using various sounds as inducer and inducee; the loudness reduction was the largest when the inducee and inducer were the same type of sounds. In Experiment 3, direct comparison with Experiments 1 and 2 was not required, but a clear demonstration of loudness reduction of the inducer was needed for testing the allocation hypothesis. Therefore, we chose tones for both the inducer and the inducee. Note that the loudness reduction of the inducer has been reported for a variety of sounds consisting of tones, noise, and speech when there were no interaural phase differences (Warren et al., 1994), so the choice of sounds is not crucial for demonstrating reallocation of some of the auditory response to the inducer over to the inducee. A 10 -msec raised cosine ramp was used at every transition between the alternating sounds, as in Experiments 1 and 2. The sound pressure level of the inducer was $70 \mathrm{~dB}$ and that of the inducee was $66 \mathrm{~dB}$. A previous diotic study (Warren et al., 1994) showed that when the level difference between the inducer and the inducee became smaller, the loudness reduction of the inducer became greater. Therefore, a small level difference is preferable to demonstrate a clear loudness reduction. However, the Warren et al. study also showed that when the inducer and inducee were matched in frequency at $1000 \mathrm{~Hz}$ (their "homophonic" condition), continuity was always perceived by all when intensity differences were $4 \mathrm{~dB}$ or more. When the level difference between the inducer and the inducee was reduced to $2 \mathrm{~dB}$, some listeners perceived an amplitude modulation of a single sound rather than TI. Therefore, we chose a 4-dB level difference in this experiment. As anticipated, all of the subjects perceived TI in the diotic (isophasic) presentation. The comparison stimulus consisted of eight pulses of a $200-\mathrm{msec}, 500-\mathrm{Hz}$ pure tone. A $10-$ msec raised cosine ramp was used as with the alternating induction stimuli, but adjacent pulses were separated by $200 \mathrm{msec}$ of silence rather than the inducee. The sound pressure level of the comparison stimulus varied between 53 and $79 \mathrm{~dB}$. The IPhD of the comparison stimulus was the same as that of the inducer in the induction stimulus under all interaural phase conditions. As a consequence, the comparison stimulus was identical to the inducer in the type of the signal, frequency, temporal pattern, and IPhD, making the loudness matching task easy. A 500-msec silent gap was introduced between the induction stimulus and the comparison stimulus. The same apparatus as that in Experiments 1 and 2 was used for the synthesis and the presentation of the stimuli, and the interaural phase relations employed in Experiments 1 and 2 were also used (MoSo, $\mathrm{M} \pi \mathrm{S} \pi, \mathrm{M} \pi \mathrm{So}$, and $\mathrm{MoS} \pi$ ).

Procedure. The loudness of the inducer was measured using the modified method-of-limits procedure described more fully in Experiment 1. The listener's task was to judge whether the comparison tone was louder than the inducer (which was also heard as pulsed). On the beginning trial of an ascending series, the comparison was presented at a low sound pressure level varying randomly from 53 to $58 \mathrm{~dB}$, and increased in 2-dB steps until the listener judged the comparison was louder than the target. For a descending series, the comparison stimulus started at a level varying randomly from 74 to $79 \mathrm{~dB}$ and then decreased in 2-dB steps until the listener judged the comparison was fainter. In addition to the four types of IPhD conditions used in Experiments 1 and 2, two baseline conditions ( $\mathrm{Bo}$ and $\mathrm{B} \pi$ ) were also examined, in which only the inducer was presented without the inducee in the induction stimulus. For $\mathrm{Bo}$, both the inducer and the comparison were cophasic; for $B \pi$, both were antiphasic. The average of the 20 series for each $\mathrm{IPhD}$ condition was taken as a detection threshold.

\section{Results and Discussion}

Figure 6 shows the mean loudness match for the inducer (the average sound pressure level of the matched comparison stimuli) for all six conditions. In the two base-

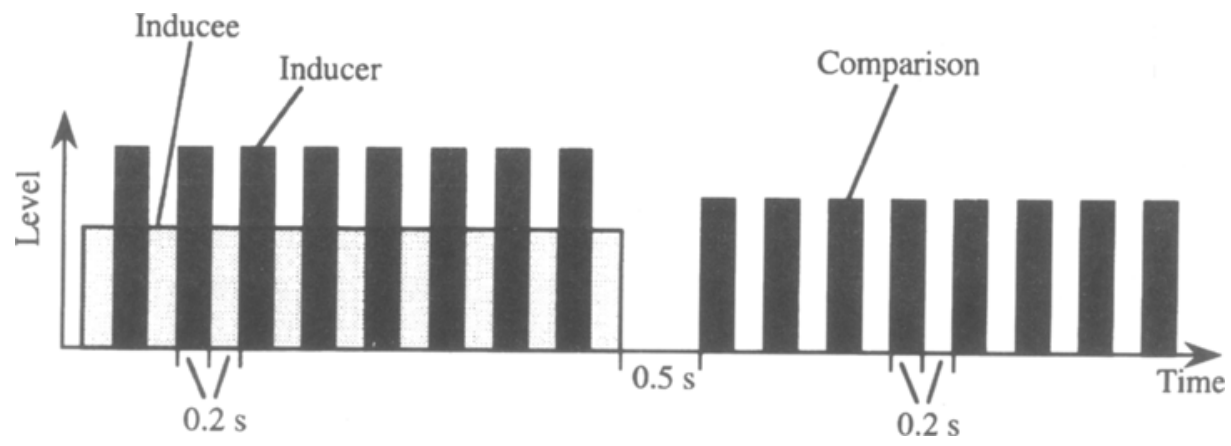

Figure 5. Stimuli employed for measuring loudness reduction of a $500-\mathrm{Hz}$ sinusoidal inducer during temporal induction. The four types of interaural phase difference combinations of inducee and inducer were $\mathrm{MoSo}, \mathrm{M} \pi \mathrm{S} \pi, \mathrm{MoS} \pi$, and $\mathrm{M} \pi \mathrm{So}$ (see text for further description). 
line conditions, the loudness (or apparent amplitude) of the 70- $\mathrm{dB}$ inducer (without the inducee) matched that of a 70-dB comparison stimulus. The standard error of matching was small, indicating a high reproducibility for loudness matching judgments with this procedure.

In MoSo and M $\pi \mathrm{S} \pi$ conditions in which the IPhD of the inducer and that of the inducee were identical, about $5 \mathrm{~dB}$ of loudness reduction was observed on average for the 5 subjects. On the other hand, in M $\pi$ So and MoS $\pi$ conditions in which the IPhD of the inducer and that of the inducee were different, as predicted by induction theory, the loudness reduction was less, averaging about $2 \mathrm{~dB}$.

A repeated measure ANOVA yielded a significant effect of IPhD conditions $[F(5,20)=10.046, p<.0005]$. Additionally, the subsequent single- $d f$ contrasts, which compared the mean of the two isophasic IPhD conditions with the mean of the two heterophasic IPhD conditions, indicated that the difference between these two groups was significant $[F(1,20)=18.941, p<.0005]$. The difference between the mean of the two heterophasic IPhD conditions and the mean of the two baseline conditions was also significant $[F(1,20)=6.990, p<.05]$.

The subjects reported that, in the isophasic IPhD conditions, the inducee was completely continuous; on the other hand, in the heterophasic IPhD conditions, continuity was not heard. However, even in the latter cases, the subjects did not perceive the two sounds as having equal durations. Some illusory lengthening of the inducee seems to have taken place, as reported for incomplete induction under diotic conditions by Warren et al. (1994).

These results indicate that IPhD relations between the inducer and the inducee affected the loudness reduction of the inducer as well as the completeness of the continuity. This is consistent with the hypothesis that TI involves a reallocation of some of the neural activity corre-

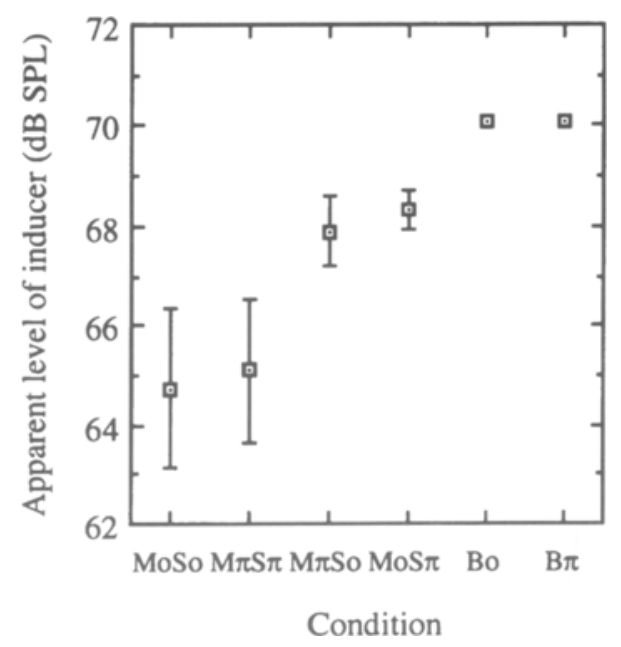

Figure 6. Loudness matches for a $500-\mathrm{Hz}$ sinusoidal inducer alternating with a 500-Hz sinusoidal inducee under four types of interaural phase difference combinations, along with two baseline conditions in which the inducee was absent. Plotted values are means and standard errors for 5 listeners. sponding to the inducer and that the extent of TI depends upon the interaural phase correspondence as well as the frequency correspondence of inducer and inducee.

However, the reallocation hypothesis does not make quantitative predictions concerning the extent of loudness reduction, and it would be inappropriate to do so. The reason is as follows: There are many factors determining loudness, such as firing rate, spread of excitation, phase lock, and so on. Among these factors, some may be affected by the reallocation, and some may not. If we assume loudness is determined by integration of these factors, it would be natural that simple calculation of loudness does not hold. Moreover, there may be individual differences in weighting such factors, resulting in individual differences of loudness reduction. We have found such individual differences in the loudness matching experiments.

\section{GENERAL DISCUSSION}

The findings from the three experiments can be summarized as follows: When the interaural phase differences (IPhDs) of the inducer and the inducee are different, (1) the upper continuity limit is lower, (2) the signal detection threshold in the presence of the background noise is lower, and (3) the loudness reduction of the inducer is smaller than when IPhDs of the inducer and the inducee are identical. In other words, temporal induction (TI) is inhibited when BMLD is large. These results suggest that some IPhD-selective processes are involved in $\mathrm{TI}$, and that the masking potential rule, which has been shown to hold in the frequency domain, also applies in the IPhD domain.

These findings are consistent with the hypothesis that TI involves a reallocation of some of the neural activity evoked by the inducer over to the inducee. The present results suggest that the extent of neural activity subject to reallocation depends upon interaural phase differences in addition to frequency relations. Physiological data support the possibility for such IPhD-selective neural activity to occur at an early stage in auditory processing, perhaps at the level of the superior olive and the inferior colliculus, which have been shown to exhibit sensitivity to interaural phase and/or time differences (Yin \& Chan, 1988).

The ecological significance of the present findings becomes evident when we think of TI as a process capable of canceling the effects of masking. Since the extent of masking depends not only upon the spectral relationship but also upon differences in azimuth of signal and masking sources giving rise to interaural acoustic differences, it is useful to employ both factors in determining whether or not the signal could be continuing. Using both factors rather than one as criteria for restoration makes TI more selective and reduces the possibility of an inappropriate perception of a signal fragment.

In considering the ecological significance of the present findings, we should keep in mind several differences between our listening conditions and conditions 
encountered outside the laboratory. First, we manipulated only IPhD to simplify the experimental situations and to keep the monaural spectral masking potential constant across the conditions. On the other hand, in the real world, differences in the spatial location of sound sources result in correlated changes in interaural time delays (a fixed time delay results in frequency-dependent phase shifts for components of broadband signals) and interaural amplitude differences (which also differ for different frequency components). Both of these factors normally change with head movements, and head movements have no effect when headphones are used. Finally, all frequencies used in the present study were within a $1 / 3-$ octave band centered at $500 \mathrm{~Hz}$, since it is well known that $\mathrm{IPhD}$ is effective in producing BMLD (Durlach \& Colburn, 1978). It is also known that IPhD has small effects on the perceived localization and on BMLD for frequencies above $1.5 \mathrm{kHz}$, presumably due to the loss of sensitivity to interaural phase differences. However, we would predict that other cues to spatial localization, such as interaural delays of a few tens of microseconds in envelope amplitude fluctuations known to produce lateralization for spectrally complex signals lacking components below $3000 \mathrm{~Hz}$ (Henning, 1974; Klumpp \& Eady, 1956; McFadden \& Pasanen, 1975), would also influence TI in a manner analogous to that observed for the interaural phase difference of low-frequency stimuli in the present study.

\section{REFERENCES}

Durlach, N. I., \& Colburn, H. S. (1978). Binaural phenomena. In E. C. Carterette \& M. P. Friedman (Eds.), Handbook of perception (Vol. 4, pp. 365-466). New York: Academic Press.

HARTMANN, W. M. (1984). A search for central lateral inhibition. Journal of the Acoustical Society of America, 75, 528-535.

HenNing, G. B. (1974). Detectability of interaural delay in highfrequency complex waveforms. Journal of the Acoustical Society of America, 55, 84-90.

Houtgast, T. (1972). Psychophysical evidence for lateral inhibition in hearing. Journal of the Acoustical Society of America, 51, 1885-1894.

KLuMPP, R. G., \& EADY, H. R. (1956). Some measurements of interaural time-difference thresholds. Journal of the Acoustical Society of America, 28, 859-860.

MCFAdDEN, D., \& PASANEN, E. G. (1975, October 24). Binaural beats at high frequencies. Science, 190, 394-396.

SODERQuisT, D. R. (1981). Binaural analysis and unmasking effects. Journal of the Acoustical Society of America, 69 (Suppl. 1), S22.

WARrEN, R. M. (1984). Perceptual restoration of obliterated sounds. Psychological Bulletin, 96, 371-383.

Warren, R. M., BashFord, J. A., JR., Healy, E. W., \& Brubaker, B. S. (1994). Auditory induction: Reciprocal changes in alternating sounds. Perception \& Psychophysics, 55, 313-322.

Warren, R. M., Obusek, C. J., \& ACKroff, J. M. (1972, June 9). Auditory induction: Perceptual synthesis of absent sounds. Science, 176, 1149-1151.

YIN, T. C. T., \& CHAN, J. C. K. (1988). Neural sensitivity to interaural time differences. In G. Edelman, W. Gall, \& W. Cowan (Eds.), Auditory function: Neurological bases of hearing (pp. 385-430). New York: Wiley.

(Manuscript received June i 7, 1994; revision accepted for publication November 4,1995 .) 